\title{
Dynamic Viscoelasticity and FT-IR Measurements of End-Crosslinking $\alpha, \omega$-Dihydroxyl Polybutadiene Solutions near the Gel Point in the Gelation Process
}

\author{
Akihiro Kolke, Norio Nemoto, ${ }^{* \dagger}$ Yuichiro Watanabe, and Kunihiro Osaki
}

\author{
Institute for Chemical Research, Kyoto University, Uji, Kyoto 611, Japan \\ * Department of Applied Physics, Faculty of Engineering, Kyushu University, \\ Hakozaki, Fukuoka 812-81, Japan
}

(Received February 29, 1996)

\begin{abstract}
Dynamic viscoelasticity of $\alpha, \omega$-dihydroxyl polybutadiene/ $p$-xylene system is studied through gelation process by end-crosslinking reaction with crosslinker which has three isocyanate groups. The gel point is determined as the reaction time $t=t_{\mathrm{c}}$, at which the storage and the loss shear moduli, $G^{\prime}(\omega)$ and $G^{\prime \prime}(\omega)$, become both proportional to $\omega^{n}$ over the whole $\omega$ range measured, where $\omega$ is the angular frequency and $n$ is a critical exponent at the gel point. The relative extent of chemical reaction $p(t)$ is estimated from a decrease in the absorption peak of the isocyanate residue by FT-IR measurement to estimate precisely $\varepsilon=\left|p(t)-p_{\mathrm{c}}\right| / p_{\mathrm{c}}$, the relative distance from the gel point $\left(p=p_{\mathrm{c}}\right)$. Effects of polymer concentration $C$ on the increase of zero shear viscosity $\eta_{0}$ before the gel point and on the increase of equilibrium modulus $G_{\text {eq }}$ after the gel point are examined in terms of critical exponents of $k$ and $z$ for power laws, $\eta_{0} \sim \varepsilon^{-k}$ and $G_{\text {eq }} \sim \varepsilon^{z}$, respectively. The exponents $n$ and $z$ are found to take universal values of $n=0.67 \pm 0.02$, and $z=2.5 \pm 0.1$ independent of $C$ in the lower concentration of $C<0.6$ where intermolecular interaction, i.e., hydrogen bonding between hydroxyl groups at chain ends are negligible. The $k$ monotonously increases with $C$, and the scaling relation among the three exponents appears only applicable in the limited $C$ range. These anomalous critical behaviors are discussed in terms of hydrogen bond coupling in the prepolymer solutions and chemical reaction scheme of this system.

KEY WORDS Dynamic Viscoelasticity / Fourier-Transform Infrared (FT-IR) / Gel Point /

End-crosslinking / Chemical Crosslinks / Gelation /
\end{abstract}

The studies on gel and gelation process have been conducted both experimentally and theoretically by a lot of researchers. In the theoretical field, Stockmayer et al. suggested a classical gel model based on the tree approximation (the classical theory). ${ }^{1,2}$ In its essential simplification, the growing cluster of reacted chains is assumed to branch off freely, the branches never being limited in their growth by the existence of other branches in the same cluster. The extent of reaction in the sol phase, $p$, is defined as the ratio of the reacted number of crosslinked point to the total number of reactable functional group.

Later, Stauffer ${ }^{3}$ and de Gennes ${ }^{4}$ pointed out that the behaviors near the critical region differed widely from the predictions of the classical theory, though the extent of reaction at the gel point, $p_{c}$, estimated by the tree approximation was found to be consistent with earlier experimental values. The divergent exponents $\gamma, v, k$, and $z$ of the weight-averaged molecular weight $M_{w}$, the radius of gyration $R_{G}$, the zero shear viscosity $\eta_{0}$, and the equilibrium modulus $G_{\mathrm{eq}}$ near the gel point are defined as

$$
\begin{array}{lll}
M_{w} \sim \varepsilon^{-\gamma} & \text { at } & p<p_{\mathrm{c}} \\
R_{\mathrm{G}} \sim \varepsilon^{-v} & \text { at } & p<p_{\mathrm{c}} \\
\eta_{0} \sim \varepsilon^{-k} & \text { at } & p<p_{\mathrm{c}} \\
G_{\text {eq }} \sim \varepsilon^{z} & \text { at } & p>p_{\mathrm{c}}
\end{array}
$$

, respectively. Here $\varepsilon$ is the relative distance from the gel point defined as

$$
\varepsilon=\left|p-p_{\mathrm{c}}\right| / p_{\mathrm{c}}
$$

\footnotetext{
† To whom correspondence should be addressed.
}

$$
\sim\left|t-t_{\mathrm{c}}\right| / t_{\mathrm{c}}
$$

where $t$ is the reaction time and $t_{\mathrm{c}}$ the corresponding time at $p_{\mathrm{c}}$. In the percolation lattice model, each lattice point is represented by a unit with a multi-functional group whose functionality is equal to the number of the nearest lattice points, and the neighboring points can form a sort of bonds. The reaction ratio corresponds to the value of $p$. The classical theory gives $\gamma=1.00$ and $v=0.50$, while the percolation theory gives 1.76 and 0.89 , respectively. ${ }^{5}$ Martin et al. interrelate the exponent $\gamma$ and $v$ to $k$ and $z$ by eq 7 and 8 using the scaling concepts. ${ }^{5-9}$

$$
\begin{gathered}
k=(\gamma+v) / 2 \\
z=3 v
\end{gathered}
$$

$k=1.33$ and $z=2.67$ are calculated using $\gamma$ and $v$ predicted by the percolation theory.

In the experimental field, considerable progress has been made on studies on the gelation systems since Winter et al..$^{10-12}$ proposed the accurate determination method of the gel point. At the gel point of $p=p_{\mathrm{c}}$, the storage and the loss moduli, $G^{\prime}(\omega)$ and $G^{\prime \prime}(\omega)$, are interrelated as

$$
\begin{gathered}
G^{\prime}(\omega) \sim G^{\prime \prime}(\omega) \sim \omega^{n} \\
\tan \delta=G^{\prime \prime}(\omega) / G^{\prime}(\omega)=\tan (n \pi / 2)
\end{gathered}
$$

where $\omega$ is the angular frequency. The power law behavior suggests that the structure of the critical gel is self-similar over a wide range of length scale. ${ }^{13-16}$ The power law of the critical gel has been found to extend over a frequency range of more than four decades, i.e., over the entire range experimentally accessible. This determination of the gel point as a critical point makes 
it possible to estimate critical exponents precisely. Then the dynamic viscoelasticity $5,6,17-31,57$ along with scattering methods ${ }^{7,32-40}$ has been widely used for studies of the critical behavior of various gelling systems. Experimental results of the critical exponents have been compared with the predictions of classical theory ${ }^{1,2,41-43}$ and those of the modern theories based on the self-similar structure of the critical gels. ${ }^{13,14,44-51}$

The experimental results reported hitherto indicate that $n$ is not constant but takes values in the range of $0<n<1$, depending on the species of gelling materials, the stoichiometric ratio, the preparation method, the molecular weight of prepolymers, and so on. Obviously the critical exponents defined near the gel point do not take the universal value. Thus it is impossible to judge what theory correctly predicts the critical behavior near the sol-gel transition and resulting gel structure.

From an experimental point of view, the nonuniversality may be partly ascribed to the effect of strong intermolecular interaction, for example entanglements, on the branched structure formed in the gelation process. This is entirely neglected in the theoretical treatment owing to mathematical complexity. Indeed Izuka et al. ${ }^{23}$ reported that the exponent $n$ decreased with increasing molecular weight of prepolymers. Since the entanglement effect on dynamics of linear flexible polymers is clearly manifested in the dramatic change in the concentration dependence of $\eta$ with dilution, it is worthwhile to study how dilution affects the critical behavior. There are three reports ${ }^{22,28,52}$ which examined the effect of dilution so far. Our previous report for the gelation process on $\alpha, \omega$-dimethyl silyl poly(propylene oxide) solutions at the balanced stoichiometric condition indicates that the critical exponents of $n, k$, and $z$ are independent of molecular weight and prepolymer concentration under the condition that entanglement coupling between prepolymers are not present. ${ }^{52}$

In this report, dynamic viscoelasticity of $\alpha, \omega$-dihydroxyl polybutadiene solutions is investigated through the gelation process by end-crosslinking to examine whether the universality for the three critical exponents holds or not for another system. A tri-functional crosslinker is used for chemical crosslinking reaction. The relative extent of reaction is directly determined using the FT-IR technique. Equation 6 is assumed to be held for crosslinking process in almost all experiments, while eq 5 is used in the theories. So we apply eq 5 to this end-crosslinking gelation system and compare experimental results of three critical exponents to percolation theory. The chemically crosslinked network by endcrosslinking is the simplest and the most appropriate system for fundamental research on the gelation process, because reactive sites are fixed along the chain and the number of reactable parts of the chain is indeed countable.

\section{EXPERIMENTAL}

\section{Materials}

The polymer used in this study is $\alpha, \omega$-dihydroxyl polybutadiene (hydroxyl-terminated polybutadiene, HTPB) kindly supplied from Japan Synthetic Rubber Co., Ltd. The number average molecular weight $M_{n}$ is

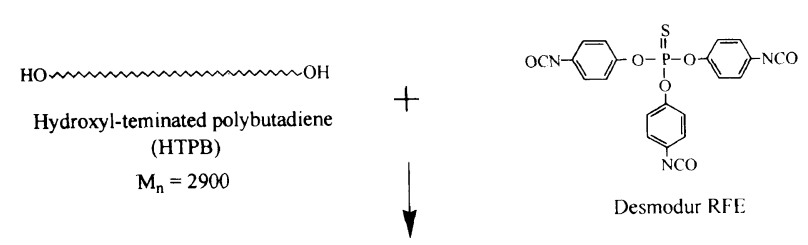

3 Dimensional Network

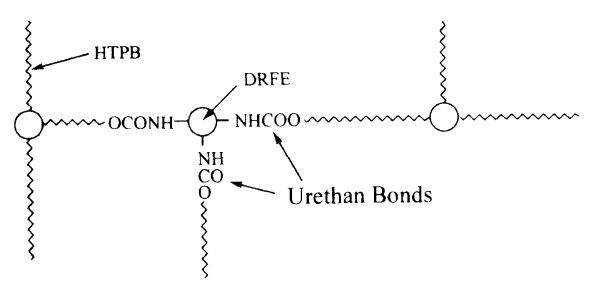

Figure 1. Reaction scheme of HTPB and DRFE in the gelation process.

2900 and the functionality is 2.15 . The bulk polymer contained about $1 \%$ of 2,2-methylene bis-(4 methyl 6 tert-butyl phenol) as antioxidant. The pure HTPB was obtained by freeze-drying in vacuum after reprecipitation. A mixture of ethylacetate and Desmodure RFE (DRFE, Bayer AG) with three isocyanate groups was dried in vacuum, and the white powder of DRFE obtained was used as crosslinker. Special grade $p$-xylene (Nacalai Tesque) was used as solvent after distillation.

Figure 1 illustrates the reaction scheme for the network formation by end-crosslinking. The hydroxyl groups of HTPB react with isocyanate groups of DREF to produce urethane bonds. One DRFE molecule has three functional groups, so that the three-dimensional network is formed in the process of end-crosslinking.

The crosslinking reaction was conducted in argon atmosphere to prevent side reaction of the isocyanate group with water molecules in the air. The solutions were prepared by dissolving, first, DRFE in $p$-xylene, adding HTPB, and then stirring rigorously at room temperature. The polymer concentration was determined by weighing, and the molar ratio of the two functional groups, $r=[\mathrm{NCO}] /[\mathrm{OH}]$, was adjusted to unity.

\section{Methods}

The storage and the loss shear moduli, $G^{\prime}(\omega)$ and $G^{\prime \prime}(\omega)$, of the samples were measured in the angular frequency $\omega$ range from 0.30 to $100 \mathrm{rads}^{-1}$ at $25.0 \pm$ $0.1^{\circ} \mathrm{C}$ using a stress-controlled rheometer (Carri-MED, CSL-100) with a $4 \mathrm{~cm}$ parallel-plate configuration covered with the humidity chamber to prevent evaporation of solvent. Time evolution of the dynamic shear modulus through the gelation process was pursued by assuming that the reaction was initiated when HTPB was added. Weight fractions $C$ of HTPB were $0.23,0.27,0.33,0.40$, $0.49,0.56,0.65$, and 0.79 .

Fourier-transform infrared (FT-IR) measurement was conducted with FT/IR-8800 (Japan Spectral Co., Ltd.) at $25.0^{\circ} \mathrm{C}$. The extent of reaction $p(t)$ was obtained from the absorption peak of the isocyanate group ( 2270 $\mathrm{cm}^{-1}$ ), from which the relative extent of reaction from the gel point $\varepsilon$ was calculated. Weight fractions of HTPB solutions measured were $0.25,0.34,0.40,0.50,0.58$, 0.66 , and 0.81 .

The intrinsic viscosity $[\eta]$ was measured by the Ub- 
belohde type viscometer at $25.0 .^{\circ} \mathrm{C}$

\section{RESULTS AND DISCUSSION}

\section{Determination of the Extent of Reaction with FT-IR Measurement}

Recently Hakiki et al. ${ }^{53}$ measured the extent of chemical reaction of $\mathrm{OH}$-terminated polystyrene with DRFE using the FT-IR technique, and reported that the chemical reaction proceeded by the second-order scheme up to $p=0.75$ which was close to the critical value at the gel point. The HTPB polymer has also one hydroxyl group at each chain end. This group reacts with the isocyanate group of the DRFE to produce the urethane bond. DRFE has three isocyanate groups, so that the three dimensional network can be formed in the reaction process. Figure 2 shows a plot of the absorbance $v s$. wave number in the range of $2100-2400 \mathrm{~cm}^{-1}$. The height of the peak at $2270 \mathrm{~cm}^{-1}$ decreased with increasing the reaction time $t$, which is ascribed to a decrease in the number of isocyanate groups in the solution. Then, $p(t)$ can be defined as

$$
p(t)=\left(h_{0}-h(t)\right) / h_{0}
$$

where $h(t)$ is the height of the peak at $t$, and $h_{0}$ is the initial peak height which is obtained by extrapolating $h$ to $t=0$.

Figure 3 shows time evolution of $1 /(1-p(t))$. The data

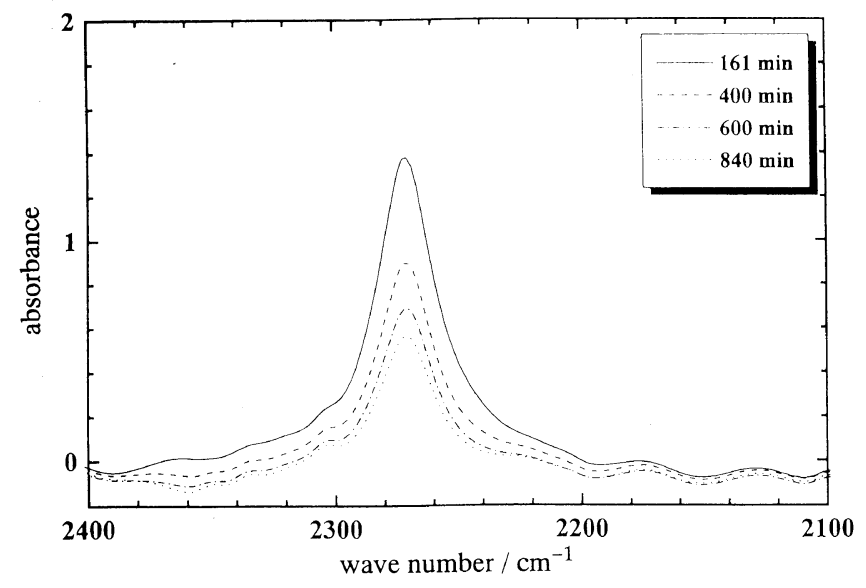

Figure 2. A plot of absorbance vs. wave number for the HTPB samples with $C=0.25$ by the FT-IR technique.

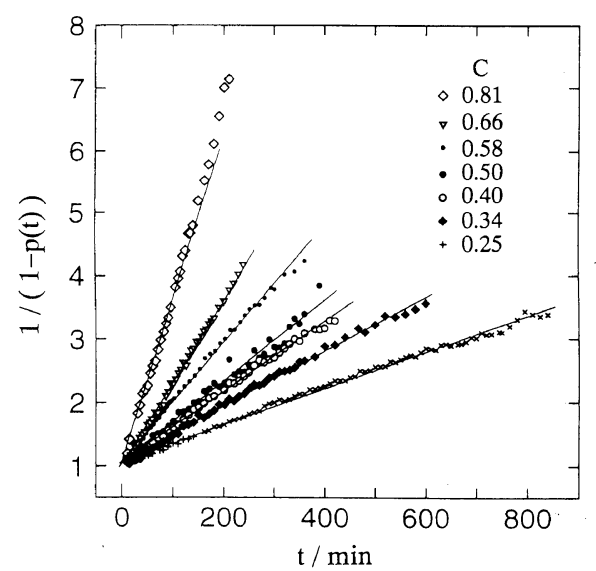

Figure 3. Time evolution of $1 /(1-p(t))$. The data could be fitted by straight lines up to $p \sim 0.75$. could be fitted by straight lines up to $p \sim 0.75$, and are represented as

$$
1 /(1-p(t))=K C_{\mathrm{NCO}}(0) t+1
$$

where $K$ is a constant and $C_{\mathrm{NCO}}(0)$ is an initial concentration of the isocyanate group which is equal to that of the hydroxyl group. This equation apparently agrees with that to be derived on the basis of the assumption that the crosslinking reaction obeys the second-order kinetics symbolically represented by $\mathrm{A}+\mathrm{B} \rightarrow \mathrm{P}$ with the reaction constant $K$, as follows. In the mean-field limit, i.e., in the reaction-limited case, the kinetics is described by

$$
-\mathrm{d} C_{\mathrm{A}}(t) / \mathrm{d} t=-\mathrm{d} C_{\mathrm{B}}(t) / \mathrm{d} t=K C_{\mathrm{A}}(t) C_{\mathrm{B}}(t)
$$

where, $C_{\mathrm{A}}(t), C_{\mathrm{B}}(t)$ are the concentrations of $\mathrm{A}, \mathrm{B}$ at reaction time $t$, respectively. Since $r=[\mathrm{NCO}] /[\mathrm{OH}]=1$ $\left(C_{\mathrm{A}}(t)=C_{\mathrm{B}}(t)\right)$, eq 13 can be easily solved in the case of $C_{\mathrm{A}}(0)=C_{\mathrm{B}}(0)$ to give eq 14 .

$$
C_{\mathrm{A}}(t)=\left(K t+1 / C_{\mathrm{A}}(0)\right)^{-1}
$$

Since $p(t)$ is defined as eq 15 ,

$$
p(t)=\left(C_{\mathrm{A}}(0)-C_{\mathrm{A}}(t)\right) / C_{\mathrm{A}}(0)
$$

We obtain eq 12 from eqs 14 and 15 .

If this reaction is really a second-order reaction, $K$ must be constant. Values of $K$ obtained by fitting of eq 12 to the data were almost constant, nearly equal to $0.13 \mathrm{~s}^{-1}$, for $C<0.55$, while it increased with increasing $C$ for $C>0.55$. The hydrogen bond between hydroxyl groups at chain ends may affect end-crosslinking chemical reaction in the higher concentrations, resulting in an increase of $K$, as is described in details in the next section in Viscosity of Prepolymer Solutions.

Equation 12 was satisfied beyond the gel point. Since the critical time $t_{\mathrm{c}}$ at the gel point is determined with dynamic viscoelastic measurement, the extent of reaction at the gel point, $p_{\mathrm{c}}$, is obtained with eq 11 and the relative extent from the gel point, $\varepsilon$, can be calculated with eq 5 .

There remains a possibility that the high reactivity of the isocyanate group causes a few types of side reactions. For example, it can react with water to produce the amine group. This amine group reacts with another isocyanate group to form the urea bond. As above stated, the crosslinking reaction proceeded in argon atmosphere, so that such the side reaction due to the presence of water was not perceived from FT-IR measurement.

\section{Viscosity of Prepolymer Solutions}

The zero shear viscosity $\eta_{0}$ of the HTPB solutions is double-logarithmically plotted against the product of the weight fraction of HTPB and its intrinsic viscosity [ $\eta]$ in Figure 4. Here $[\eta]$ was obtained from the Huggins, the Fuoss-Mead, and the Billmeyer plots as $13.7 \mathrm{~cm}^{3}$ $\mathrm{g}^{-1}$, nearly equal to the literature values. ${ }^{54}$ The overlapping concentration $C^{*}$ was calculated using $C^{*}=$ $1 /[\eta] \rho_{\mathrm{s}}$ as 0.085 by weight fraction, where $\rho_{\mathrm{s}}$ is the density of the solvent. The $C^{*}$ value suggests that HTPB chains are overlapped in the concentration range where the gelation process was studied. On the other hand, the critical molecular weight $M_{\mathrm{c}}$ for bulk 1,4-polybutadiene 


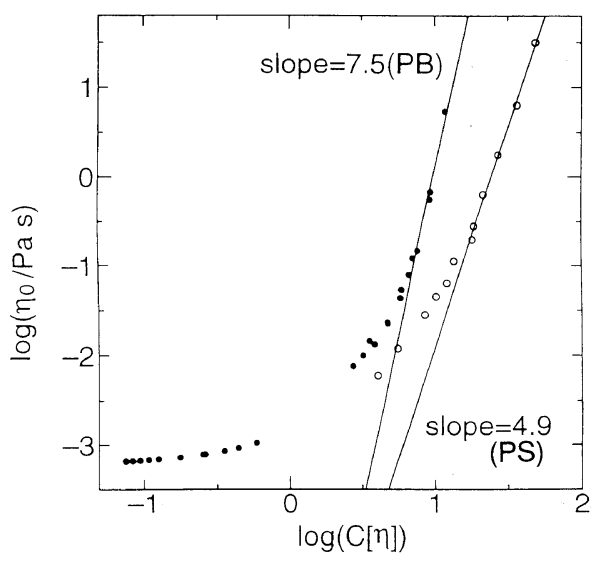

Figure 4. The zero shear viscosity $\eta_{0}$ of the HTPB solutions is plotted against the product of the weight fraction $C$ of HTPB and its intrinsic viscosity $[\eta]$.

(about $50 \%$ cis) is reported ${ }^{55}$ as 5900 and the configuration of the HTPB samples used in this study is a trans-rich one from NMR measurements. Therefore, the prepolymer solutions might be slightly entangled only at very high $C$. Nevertheless, there is a remarkable change in the slope of $\eta_{0}$ from 2.6 to 7.5 at relatively low $C$ of $C[\eta] \sim 0.5$.

Figure 4 also shows the data of polystyrene (PS) of the molecular weight of 300000 in toluene at $40^{\circ} \mathrm{C}$, where strong intermolecular interaction is supposed to be negligible. ${ }^{56}$ The slope in the higher concentration for the HTPB solution, 7.5, was larger than the corresponding slope of 4.9 for the PS solution, and a steep increase in $\eta_{0}$ took place at lower $C[\eta]$ for the former solution. Thus we are led to consider that another type of intermolecular interaction may exist between HTPB polymers. It seems natural to suppose that this interaction may be the hydrogen bonding, because the HTPB polymer has hydroxyl groups at chain ends.

In order to get qualitative evidence to the above supposition, we conducted IR measurement. In Figure 5, the transmittance is plotted against wave number from 1000 to $4000 \mathrm{~cm}^{-1}$ for $C=0,0.20,0.50,0.60,0.70,0.80$, $0.90,1.0$. The peak at $3584 \mathrm{~cm}^{-1}$ is the peak of the hydroxyl group without hydrogen bond, which was observed for all solutions. The broad peak at $3353 \mathrm{~cm}^{-1}$ can be assigned as the peak of the hydrogen bonded $\mathrm{OH}$ group, which became appreciable above $C=0.6$ and becomes larger with increasing $C$. Therefore the hydrogen bonds between the hydroxyl groups at chain ends, being surely present at $C>0.6$, is likely to affect the $C$ dependence of the zero shear viscosity. It is probable that the hydrogen bonds may also affect the reaction rate constant $K$. Consequently, this effect is considered to give serious influence on the critical behavior of the solutions near the gel point especially at higher $(C>0.6)$ concentrations.

\section{Determination of the Gel Point}

Figure 6 shows changes in the frequency dependence of $G^{\prime}$ and $G^{\prime \prime}$ of the HTPB solution at $C=0.49$ as end-crosslinking of polymer chains proceeds by the tri-functional crosslinker DRFE. The data are shifted along the horizontal axis by a factor of $10^{\mathrm{b}}$ in order to

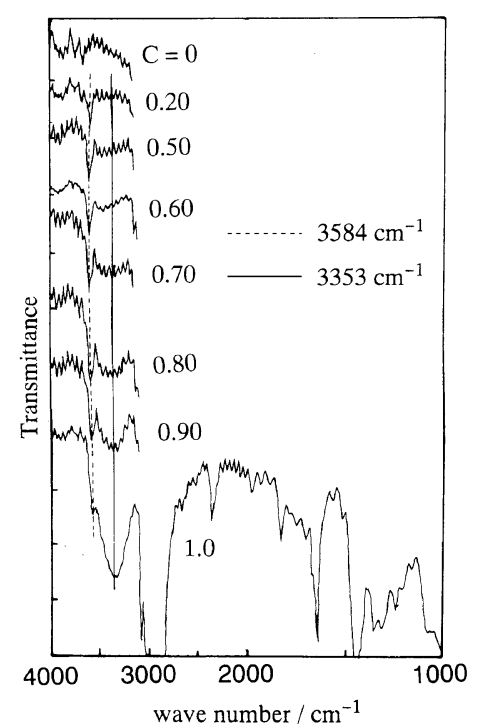

Figure 5. A plot of transmittance $v$. wave number with the FT-IR measurement.

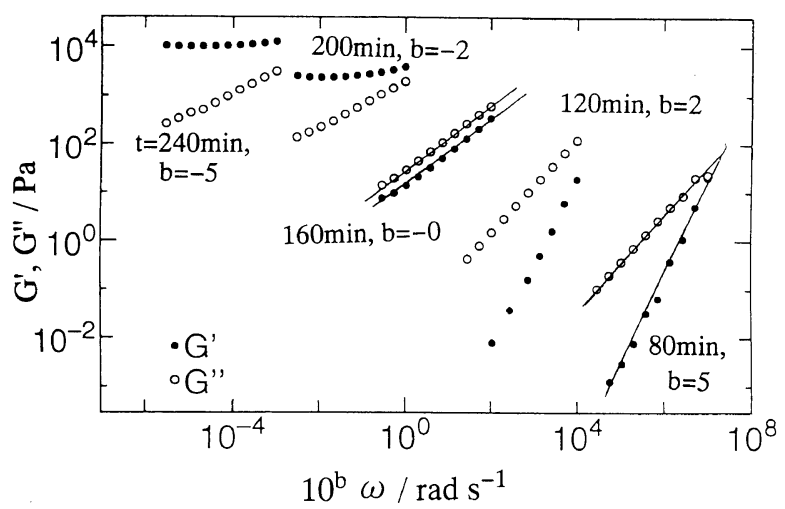

Figure 6. Angular frequency dependence of $G^{\prime}(\omega)$ and $G^{\prime \prime}(\omega)$ of five HTPB solutions with $C=0.49$ at $t=80,120,160,200$, and $240 \mathrm{~min}$. The data are shifted to avoid overlapping of data points by $10^{\mathrm{b}}$ as indicated in the figure.

avoid data overlapping. At $t=80$ and $120 \mathrm{~min}, G^{\prime}$ and $G^{\prime \prime}$ were proportional to $\omega^{2}$ and $\omega^{1}$ over the measured range, respectively, indicating that the sample remained a viscous fluid. The viscoelastic behavior of the sample rather abruptly changed as the gel point was approached. At the gel point $\left(t=t_{\mathrm{c}}\right), G^{\prime}$ and $G^{\prime \prime}$ should become both proportional to $\omega^{n}$ over the whole range. ${ }^{10,11}$ The data at $t=160 \mathrm{~min}$, being very close to the gel point, appear to show such viscoelastic response. However a time span of the one consecutive measurement over a $\omega$ range of $0.3-100 \mathrm{rads}^{-1}$ was usually about three minutes, in which the extent of chemical reaction at the first data point was different from that at the last data point. Since the viscoelastic behavior sharply changes in the critical region, the power law behavior can not be directly observed from this type of the viscoelastic measurement in a strict sense. The data at $t=200$ and 240 min show that the sample was beyond the critical gel point. A plateau appeared in the $G^{\prime}$ curve after $t_{\mathrm{c}}$ at the low $\omega$ end, and the gel can be characterized by the equilibrium modulus $G_{\text {eq }}$.

As stated in Introduction, the gel point or $t_{\mathrm{c}}$ is more accurately determined from examination of time evolution of $\tan \delta\left(=G^{\prime \prime} / G^{\prime}\right)$ at various frequencies, since 


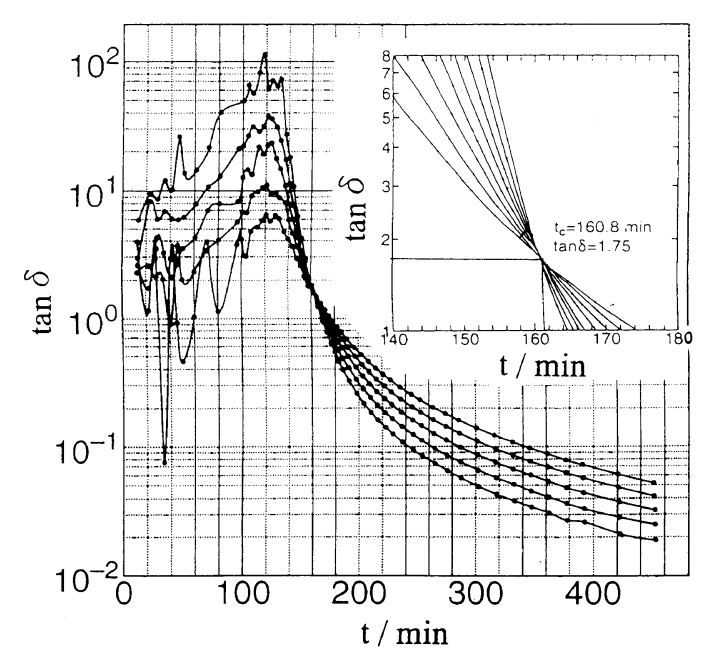

Figure 7. A plot of $\tan \delta$ against the reaction time $t$ for the sample with $C=0.49$. The five curves obtained at $\omega=7.7,14,28,52$, and 100 $\mathrm{rad} \mathrm{s}^{-1}$ meet at one point. The inset is magnification of data points neat $t_{\mathrm{c}}$.

the chemical reaction time $t$ is calculable for each data point. Figures 7 gives a plot of $\tan \delta$ against $t$ for the sample with $C=0.49$. The proportionality of $G^{\prime}$ and $G^{\prime \prime}$ to $\omega^{n}$ means that $\tan \delta$ must be independent of $\omega$, as shown with eq 9 and 10 . Evidently, the five curves obtained at $\omega=7.7,14,28,52,100 \mathrm{rad} \mathrm{s}^{-1}$ meet at one point. Thus this type of plot provides more accurate determination of the gel point as the crossing point, and accordingly of $n$ and $t_{\mathrm{c}}$. The same is true at all concentrations. Values of $n$ and $t_{\mathrm{c}}$ thus determined are given in Table I. The time dependence of $\tan \delta$ in Figure 7 suggests that viscoelastic properties of the system through the gelation process can be separately discussed by dividing the curves into three regions; the region 1 where $\tan \delta$ gradually increases with reaction time $t$ and reaches a maximum; the region 2 where $\tan \delta$ after the maximum decreases with $t$, rapidly at the higher $\omega$ to the gel point; the region 3 beyond the gel point. In the region 1, all the solutions including the melt were essentially viscous Newtonian fluids ${ }^{55}$ so that the behavior of $G^{\prime} \sim \omega^{2}$ and $G^{\prime \prime} \sim \omega^{1}$ were clearly observed, and the zero shear viscosity $\eta_{0}$ gradually increased with $t$. In the region 2, the solutions were not viscous fluids any longer. In addition to rapid increase of $\eta_{0}$ compared to that in the region 1, the solutions became more and more elastic, i.e., $G^{\prime}$ increased more rapidly than $G^{\prime \prime}$, which is reflected as the decrease in $\tan \delta$ in accordance with formation of large clusters. In the region 3 , the system was percolated, and $G_{\text {eq }}$ became larger and larger with the reaction time, indicating further development of a three dimensional network structure. The critical region is included in the regions 2 and 3.

Figure 8 gives a plot of the exponent $n$ against polymer weight fraction $C$. The $n$ value took the smallest value of 0.54 at $C=0.79$. It increased with decreasing $C$, and was independent of $C$ in $C<0.6$, equal to $0.67 \pm 0.02$. In our previous experimental result on gelation of end-crosslinking poly(propylene oxide) solutions, ${ }^{52}$ we found that $n$ took a constant value of $0.67 \pm 0.01$ irrespective of molecular weight and concentration of prepolymer solutions whenever they were not entangled, and $n$ decreased down to 0.51 with increasing entangle-

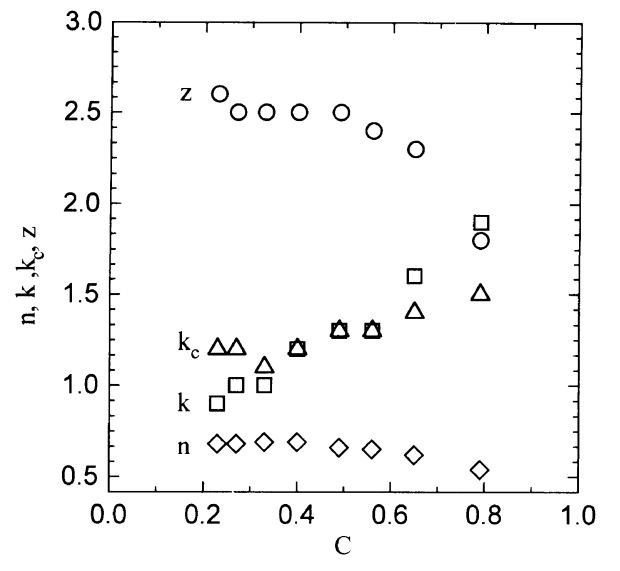

Figure 8. Critical exponents of $n, k, k_{\mathrm{c}}$, and $z$ are plotted against weight fraction $C$ of HTPB.

ment density. The small $n$ value of 0.54 may be due to the intermolecular interactions such as hydrogen bonding at chain ends. The $n$ took a universal constant of $0.67 \pm 0.02$ in the absence of couplings between prepolymers before reaction.

\section{Critical Region near the Gel Point}

Since IR and DVE measurements were separately done for HTPB solutions with $C$ slightly different from each other, the value of $\varepsilon$ at reaction time $t$ for respective solutions used for gelation study was calculated by the following procedure. In a plot of $K C_{\mathrm{NCO}}(0) v s . C$, the data point could be fitted by a straight line, expressed as

$$
\log K C_{\mathrm{NCO}}(0)=-6.8+3.8 C
$$

In substitution of eq 12 and 16 to 15 , we obtain eq 17 for $\varepsilon$,

$$
\varepsilon=\frac{t\left(K C_{\mathrm{A}}(0) t_{\mathrm{c}}+1\right)}{t_{\mathrm{c}}\left(K C_{\mathrm{A}}(0) t+1\right)}-1
$$

Beyond the gel point, the largest cluster is fully developed from one side to the other side of the sample volume, so that the system may be characterized by the equilibrium modulus $G_{\text {eq }}$ to be estimated as the plateau value at the low $\omega$ end in the $G^{\prime}(\omega) v s . \omega$ plot. As stated in Introduction, the critical gel can be discussed in terms of the two exponents $k$ and $z$ appearing in eq 3 and 4 . The $\varepsilon$ dependence of $G_{\text {eq }}$ is shown for eight solutions with $C$ from 0.23 to 0.79 double-logarithmically in Figure 9 where $\varepsilon$ is multiplied by $10^{\mathrm{b}}$ to avoid data overlapping. A straight line was successfully drawn to data of each solution, from which the exponent $z$ was estimated. It should be noted that the last data point with the smallest $\varepsilon$ at each concentration was taken from a $G^{\prime}$ curve which showed very slight $\omega$ dependence even at the low $\omega$ end, so that $G_{\text {eq }}$ is smaller than the value shown in the figure. Values of $z$ thus determined are listed in Table I and also plotted against $C$ in Figure 8. In the case where the entanglement or the hydrogen bond coupling was not present for the prepolymer solutions, i.e., $C<0.6, z$ was equal to $2.5 \pm 0.1$, independent of $C$. The percolation theory predicts the three critical exponents, $n=0.67, z=2.67$, and $k=1.33 .{ }^{5}$ The obtained value of $z$ is smaller but not far from the theoretical value. On the other hand, $z$ became smaller for $C>0.6$ 


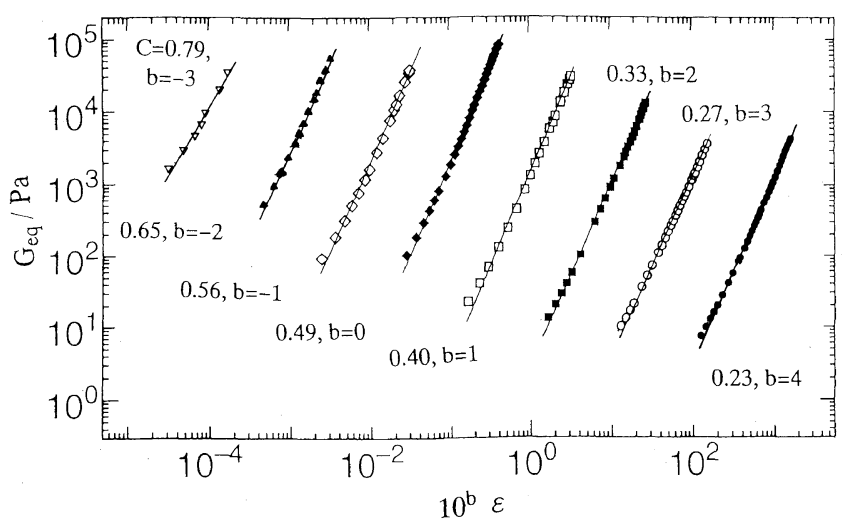

Figure 9. The $\varepsilon$ dependence of $G_{\text {eq }}$ is shown on eight solutions with $C$ from 0.23 to 0.79 , where $\varepsilon$ is multipled by $10^{\mathrm{b}}$ to avoid data overlapping. The exponent $z$ is estimated as the slope of straight lines fitted to respective data in the critical region.

Table I. Experimental results of end-crosslinking HTPB system

\begin{tabular}{|c|c|c|c|c|c|c|c|}
\hline C & $t_{\mathrm{c}} / \mathrm{min}$ & $n$ & $z$ & $k$ & $k_{\mathrm{c}}$ & $z_{\mathrm{t}}$ & $k_{\mathrm{t}}$ \\
\hline & & & & & & & \\
\hline & $582 \pm$ & & & & & & \\
\hline & 364 & & & & & & \\
\hline & 229 & & & & & & \\
\hline 49 & 161 & 0.66 & & & & & \\
\hline 56 & 127 & 0.65 & $2.4 \pm 0.1$ & $1.3 \pm 0.1$ & $1.3 \pm$ & $2.0 \pm$ & \\
\hline 65 & $87.5 \pm 0.5$ & 0.62 & $2.3 \pm$ & $1.6 \pm$ & 1.4 & $2.0 \pm$ & $1.8 \pm$ \\
\hline .79 & $71.3 \pm 0$ & 0.54 & $1.8 \pm 0.1$ & $1.9 \pm 0.1$ & $1.5 \pm 0.1$ & $1.7 \pm 0.1$ & $2.2 \pm 0.2$ \\
\hline
\end{tabular}

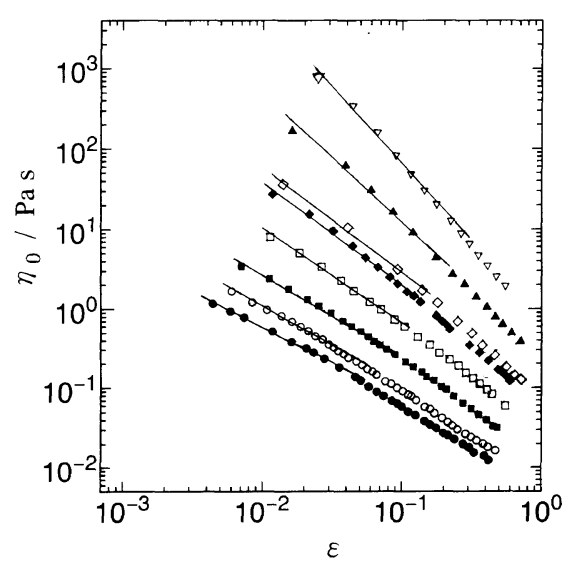

Figure 10. The $\varepsilon$ dependence of $\eta_{0}$ is shown on eight solutions with $C$ from 0.23 to 0.79 . Symbols for $C$ are the same as in Figure 9. The exponent $k$ is estimated as the slope of straight lines fitted to respective data belonging to the region 2 described in the text.

than the constant value at lower $C$. The decrease of $z$ is tentatively attributed to the increase of hydrogen bonding.

Figure 10 shows the $\varepsilon$ dependence of the zero shear viscosity $\eta_{0}$ for eight solutions with $C$ from 0.23 to 0.79 In order to estimate $k$, the data were fitted by straight lines using data points belonging to the region 2. As is clear from the figure, the critical region appears to shift to smaller $\varepsilon$ values with increasing dilution. The estimated $k$ values are summarized in the Table I and are also plotted against $C$ in Figure 8 . The $k$ did not take a constant value even in the lower concentrations but monotonously increased with increasing $C$. Assuming that three exponents satisfy the prediction of the scaling theory (18),

$$
n=z /(k+z)
$$

we calculated the value of $k_{\mathrm{c}}$ using experimental values of $n$ and $z$, and plot them in Figure 8. The $k$ and $k_{\mathrm{c}}$ is in good agreement with each other in the intermediate concentration range of $0.30<C<0.60$, in contrast to the disagreement in the lower and the higher concentration ranges, i.e., the scaling relation (18) appears to hold only in the limited $C$ range. The discrepancy in the higher $C$ range might be attributable to the increase in number of hydrogen bonds, but another type of reasoning is likely to be needed for the disagreement in the lower $C$ range.

In the case of poly(propylene oxide) (PPO) solution, ${ }^{52}$ the crosslinking reaction proceeded by the formation of the siloxane bond between two chains after the hydrolysis of the dimethyl silyl groups at chain ends in the presence of water and catalyst. Because the association of polymers by the hydrogen bond was not present, the critical behavior was controlled only by entanglement coupling, so that universal values were obtained for the three exponents as $n=0.67 \pm 0.02, k=1.0 \pm 0.1$, and $z=2.0 \pm 0.1$ in the absence of entanglements for prepolymer solutions. On the other hand, in the HTPB gelation system, entanglement couplings hardly affect the critical behavior because of low $M$ of the HTPB sample used, but the intermolecular association due to hydrogen bonding between hydroxyl groups for the prepolymer solutions (Figures 4 and 5) seriously affected the three exponents in the higher concentration region. In the lower concentrations where the extent of association is small, we expect that the HTPB solutions may exhibit the universal critical behavior as found for the PPO system. Indeed, $n$ and $z$ took constant values, but $k$ did not, and the scaling relation was inapplicable in the lower $C$.

There is also a difference in absolute magnitude of $z$ between the two systems. The universal value of $z$ for the end-crosslinking PPO system without entanglement couplings $^{52}$ was obtained as $2.0 \pm 0.1$ using eq 6 for an estimate of $\varepsilon$, which is smaller than $z=2.5 \pm 0.1$ of HTPB where eq 5 was used for an estimate of $\varepsilon$. In order to examine to what extent the exponent $z$ is altered by the use of eq 6 , we replotted $G_{\text {eq }}$ of the HTPB solutions against $\varepsilon_{\mathrm{t}}=\left|t-t_{\mathrm{c}}\right| / t_{\mathrm{c}}$. Values of $z_{\mathrm{t}}$ obtained are listed in Table I, and are equal to $2.1 \pm 0.1$ except the one at the highest $C$ and close to the value of the PPO system. Equation 6 was also used to estimate $k_{\mathrm{t}}$ from a plot of $\eta$ vs. $\varepsilon_{t}$ given in Figure 11, which are listed in Table I. The difference between $k_{\mathrm{t}}$ and $k$ is quite small in the low $C$ and also their magnitudes are close to the $k$ value obtained for the PPO system, though $k_{\mathrm{t}}$ still showed a tendency to increase monotonously from 0.9 to 2.2 with increasing $C$ as $k$. In comparison with Figures 10 and 11 , it should be noticed that the use of $\varepsilon$ permitted, in appearance, viscosity measurements in the critical region more deeply by about a factor of $2-3$ than the use of $\varepsilon_{t}$. This was found also true for the critical behavior of $G_{\text {eq }}$. These rather confusing results give a serious doubt about either the use of $\varepsilon_{\mathrm{t}}=\left|t-t_{\mathrm{c}}\right| / t_{\mathrm{c}}$ as the measure of the relative extent of chemical reaction for the chemically gelling system or if $\varepsilon$ was accurately determined for the HTPB system, or about the both. However, the follow- 


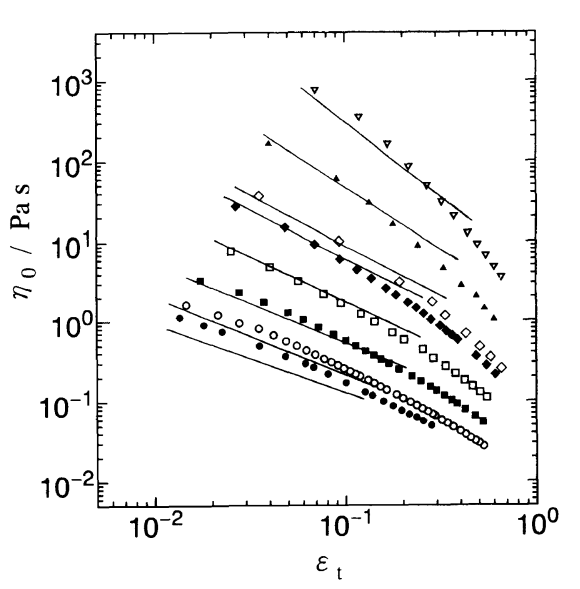

Figure 11. $\eta_{0}$ data given in Figure 10 is plotted against $\varepsilon_{1}$. Straight lines drawn in Figure 10 are replotted against $\varepsilon p_{c} /\left(p_{c}-1 / 3\right)$ in the figure.

ing discussion, though not quantitative, will lead to a reasonable conclusion that the difference in the chemical reaction scheme between the two systems resulted in different values of the critical exponents to the respective systems.

In the PPO system, every formation of the siloxane bond after the hydrolysis of the dimethyl silyl groups contributes to growth of clusters or of the three dimensional network, i.e., give rise to chain connectivity excluding cyclization. If the chemical reaction in the critical region might successively occur within displacement of chains in clusters comparable to the end-to-end distance of the primary chain, then, $\varepsilon=\varepsilon_{\mathrm{t}}$ would be considered as plausible assumption. This is not the case for the HTPB system, to which the reaction of a free crosslinker DRFE with three isocyanate $(\mathrm{CN})$ groups with a hydroxyl $(\mathrm{OH})$ group at one chain end merely creates an active chain for further crosslinking, and the reaction of its second $\mathrm{CN}$ group with another HTPB chain is able to contribute to chain connectivity necessary for percolation. In an extreme case that the first reaction of one of the three $\mathrm{CN}$ groups with the $\mathrm{OH}$ group were supposed to be very rapid, we would have, at the extent of chemical reaction $p=1 / 3$, a mixture of linear HTPB chains with either two or one $\mathrm{CN}$ groups at chain ends and without the $\mathrm{CN}$ group whose molar ratios are statistically $1: 2: 3$ under our experimental condition of $r=[\mathrm{NCO}] /[\mathrm{OH}]=1$, and completion of bimolecular reaction among these three types of chains gives $p=7 / 12$. This may explain to some extent why the gelation process of the HTPB system apparently followed the second-order kinetics up to high $p$. Then, the relative exponent of chemical reaction related to chain connectivity, $\varepsilon^{\prime}$, is not given by eq 5 but must be calculated as $\left|p-p_{\mathrm{c}}\right| /\left(p_{\mathrm{c}}-1 / 3\right)$ or $\varepsilon p_{\mathrm{c}} /\left(p_{\mathrm{c}}-1 / 3\right)$ using $p$ and $p_{\mathrm{c}}$ values determined from IR. In order to test that this conjecture may explain the large difference between $\varepsilon$ and $\varepsilon_{\mathrm{t}}$ at the same chemical reaction time, we estimated $p_{\mathrm{c}}$ as follows.

The critical time $t_{\mathrm{c}}$ listed in Table I was proportional to $C^{-2.0 \pm 0.1}$ in the $C$ range measured. The relation of $t_{\mathrm{c}}$ and $C$ was described by

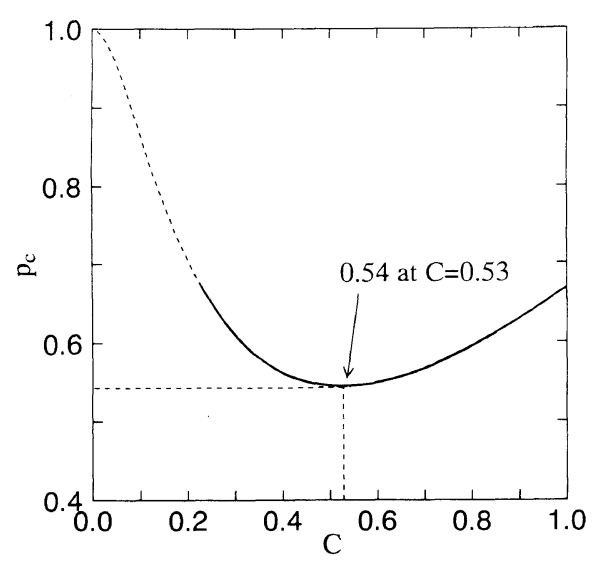

Figure 12. A plot of the extent of reaction at the gel point $p_{\mathrm{c}} v s$. weight fraction $C$ of the HTPB solutions following eq 20 .

$$
\log \left(t_{\mathrm{c}}\right)=3.7-2.0 \log C
$$

Equations 12, 16, and 19 lead to a result that $p_{\mathrm{c}}$ is represented to a good approximation as

$$
\begin{gathered}
p_{\mathrm{c}}=e^{x} /\left(1+e^{x}\right) \\
x=-3.1+3.8 C-2.0 \log C
\end{gathered}
$$

As shown in Figure $12, p_{\mathrm{c}}$ is not independent of $C$, but decreases with increasing $C$ in the lower concentration, takes a minimum value of 0.54 at $C=0.53$, and increases with increasing $C$. With these $p_{\mathrm{c}}$ values, $\varepsilon^{\prime}$ can be easily calculated, and $\eta$ vs. $\varepsilon^{\prime}$ curves of respective solutions are obtained by shifting corresponding $\eta v s . \varepsilon$ curves in Figure 10 along the horizontal axis to the right by $p_{\mathrm{c}} /\left(p_{\mathrm{c}}-1 / 3\right)$. It is to be noted that this shift does not alter the $k$ value. The straight lines in Figure 10 after conversion of the variable $\varepsilon$ to $\varepsilon^{\prime}$ are reproduced in Figure 11. All lines now look to represent closely the dependence of $\eta$ on $\varepsilon_{\mathrm{t}}$ in the critical region especially in the high $C$ range except the highest $C$ sample. Thus, the conjecture concerning the extent of chemical reaction seems successful in explanation of the observed difference in magnitude between $\varepsilon$ and $\varepsilon_{\mathrm{t}}$ at the same chemical reaction time. In fact, it may be noted that eq 17 gives a following relation between $\varepsilon$ and $\varepsilon_{\mathrm{t}}$,

$$
\varepsilon=\varepsilon_{\mathrm{t}} /\left(K C_{\mathrm{A}}(0) t+1\right)
$$

Near $t \sim t_{\mathrm{c}}, K C_{\mathrm{A}}(0) t+1 \sim K C_{\mathrm{A}}(0) t_{\mathrm{c}}+1=1 /\left(1-p_{\mathrm{c}}\right)$. Equating $p_{\mathrm{c}} /\left(p_{\mathrm{c}}-1 / 3\right)$ to $1 /\left(1-p_{\mathrm{c}}\right)$, we obtain $p_{\mathrm{c}}=0.58$ in consistence with the values in the high $C$ range shown in Figure 12. We arrive to the same conclusion that $\varepsilon$ had better to be replaced by $\varepsilon^{\prime}$, when the logarithmic plot of $G_{\text {eq }} v s$. $\varepsilon$ in Figure 9 is compared with the corresponding $G_{\text {eq }} v s$. $\varepsilon_{\mathrm{t}}$ plot.

The deviation in the lower $C$ and at the highest $C$ is partly ascribed to the simple assumption that all DRFE molecules reacted before entering the critical region, and partly to that crosslinking reaction in the HTPB system with three different types of linear chains is more complicated than that in the PPO system. We can not deny that, in reality, free DRFE molecules and big clusters would be coexistent even near the gel point. We speculate furthermore that cyclization of a primary 
chain due to chemical reaction between one $\mathrm{CN}$ group at one end and one $\mathrm{OH}$ group at another end may occur more frequently with increasing dilution. This type of reaction increases $p$ but the bond formed is useless for growth of clusters, which may lead to a larger $p_{\mathrm{c}}$ value as well as a smaller increase in viscosity with $\varepsilon$ leading to smaller $k$ as observed. In the highly concentrated region, on the other hand, hydrogen bonding clearly helps formation of intermolecular bonding, so that the system may enter the critical region at smaller $p$, i.e., at larger $\varepsilon$ with the larger exponent $k$. Thus, monotonous increase of $k$ with $C$ is tentatively interpreted as being due to the combined effects of intramolecular cyclization at low $C$ and hydrogen bonding at high $C$. As mentioned by Takahashi et al., ${ }^{57}$ the exponents $n$ and $z$ are determined by the backbone structure of the percolation cluster. Therefore the cyclization effect in the low $C$ affects these two exponents to lesser extent, resulting in that they take constant values in the $C$ range where the extent of association due to hydrogen bonding is negligible.

The $p_{\mathrm{c}}$ at $C=1.0$ was calculated from eq 20 to be 0.67 . As noted in Introduction, the classical theory developed by Flory, and Stockmayer et al. was based on the tree approximation not including the intramolecular cyclic reaction and the steric hindrance. The theory gives

$$
r^{\prime} p_{\mathrm{c}}^{2}=1 /(f-1)(g-1)
$$

where $r^{\prime}$ is the ratio of the total number of reactive groups in crosslinkers to the corresponding number in polymers, $f$ the number per one crosslinker, and $g$ the number per one polymer. Calculation with eq 23 gave $p_{\mathrm{c}}=0.707$, which is apparently close to 0.67 . However, the theoretical predictions of $k=0.75$ and $z=1.5$ are very different from experimental values.

The percolation theory introduced by Stauffer et al. ${ }^{45}$ explains the fractal structure in terms of the percolating clusters, and the computer simulation method is utilized to estimate the critical exponent. The percolation is classified as a site percolation, a bond percolation, and a site-bond percolation. The results are dependent on the spatial dimension, the model lattice, and the definition of clusters. In the treatment that the cluster is formed by the nearest lattice point with the bond percolation, the simulated value of $p_{c}$ is 0.388 in the diamond lattice, 0.249 in the simple cubic, 0.179 in the body center cubic, and 0.119 in the face center cubic for the spatial dimension of 3 . The experimental results $p_{\mathrm{c}}$ for which even the minimum value is 0.54 are larger than these values, but values of $\left(p_{c}-1 / 3\right)$ ranging from 0.21 to 0.33 are within simulated values.

Acknowledgments. We are grateful to Professors T. Miyamoto and T. Fukuda, and Dr. Y. Tsujii, Institute for Chemical Research, Kyoto University, for their advice and discussion on FT-IR measurement. We also thank Japan Synthetic Rubber Co. for gift of the HTPB sample.

\section{REFERENCES}

1. W. H. Stockmayer, J. Chem. Phys., 11, 45 (1943).
2. W. H. Stockmayer, J. Chem. Phys., 12, 125 (1944).

3. D. Stauffer, Faraday Trans. II, 72, 1354 (1976).

4. P.-G. de Gennes, J. Phys. (Paris) Lett., 37L, 61 (1976).

5. D. Adolf, J. E. Martin, and J. P. Wilcoxon, Macromolecules, 23, 527 (1990).

6. J. E. Martin, D. Adolf, and J. P. Wilcoxon, Phys. Rev. Lett., 61, 2620 (1988)

7. J. E. Martin and K. D. Keefer, Phys. Rev. A, 34, 4988 (1986).

8. J. E. Martin, J. P. Wilcoxon, and D. Adolf, Phys. Rev. A, 36, 1803 (1987).

9. J. E. Martin, D. Adolf, and J. P. Wilcoxon, Phys. Rev. A, 39, 1325 (1989).

10. F. Chambon and H. H. Winter, Polym. Bull., 13, 499 (1985).

11. H. H. Winter and F. Chambon, J. Rheol., 30, 367 (1986).

12. H. H. Winter, "Encyclopedia of Polymer Science and Engineering," Supplement Volume, 2nd ed, John Wiley \& Sons, Inc., New York, N.Y., 1989.

13. M. Muthukumar, J. Chem. Phys., 83, 3161 (1985).

14. M. Muthukumar and H. H. Winter, Macromolecules, 19, 1285 (1986).

15. M. E. Cates, J. Phys., 46, 1059 (1985).

16. T. Vilgis and H. H. Winter, Progr. Colloid Polym. Sci., 266, 494 (1988).

17. F. Chambon, Z. S. Petrovic, W. J. MacKnight, and H. H. Winter, Macromolecules, 19, 2146 (1986).

18. F. Chambon and H. H. Winter, J. Rheol., 31, 683 (1987).

19. H. H. Winter, Progr. Colloid Polym. Sci., 75, 104 (1987).

20. H. H. Winter, P. Morganelli, and F. Chambon, Macromolecules, 21, 532 (1988).

21. E. M. Valles, J. M. Carella, H. H. Winter, and M. Baumgaertel, Rheol. Acta, 29, 535 (1990).

22. J. C. Scanlan and H. H. Winter, Macromolecules, 24, 47 (1991).

23. A. Izuka, H. H. Winter, and T. Hashimoto, Macromolecules, 25, 2422 (1992)

24. M. Adam, M. Delsanti, and D. Durand, Macromolecules, 18, 2285 (1985).

25. D. Durand, M. Delsanti, M. Adam, and J. M. Luck, Europhys. Lett., 3, 297 (1987).

26. D. Lairez, M. Adam, J. R. Emery, and D. Durand, Macromolecules, 25, 286 (1992).

27. D. F. Hodgson and E. J. Amis, Macromolecules, 23, 2512 (1990).

28. R. Muller, E. Gerard, P. Dugand, P. Rempp, and Y. Gnanou, Macromolecules, 24, 1321 (1991).

29. M. Antonietti, K. J. Folsch, H. Sillescu, and Pakula, T. Macromolecules, 22, 2812 (1989).

30. D. Durand, F. Naveau, J. P. Busnel, M. Delsanti, and M. Adam, Macromolecules, 22, 2011 (1989).

31. M. Adam, M. Delsanti, and J. P. Munch, Physica A, 163, 85 (1990).

32. M. Schmidt and W. Burchard, Macromolecules, 14, 370 (1981).

33. F. Schosseler and L. Leibler, J. Physique Lett., 45, 501 (1984).

34. J. E. Martin, J. P. Wilcoxon, and D. Adolf, Phys. Rev. A, 36, 1803 (1987).

35. J. E. Martin and J. P. Wilcoxon, Phys. Rev. Lett., 61, 373 (1988).

36. G. Dietler, C. Aubert, D. S. Cannell, and P. Wiltzius, Phys. Rev. Lett., 57, 3117 (1986).

37. M. Adam, M. Delsanti, J. P. Munch, and D. Durand, Phys. Rev. Lett., 61, 706 (1988).

38. R. Vacher, T. Woignier, J. Pelous, and E. Courtens, Phys. Rev. $B, 37,6500$ (1988).

39. E. V. Patton, J. A. Wessen, M. Rubinstein, J. C. Wilson, and L. E. Oppenheimer, Macromolecules, 22, 1946 (1989).

40. F. Ferri, B. J. Frisken, and S. Cannell, Phys. Rev. Lett., 67, 3626 (1991).

41. See, for example: P. J. Flory, "Principles of Polymer Chemistry," Cornell University Press, Ithaca, New York, 1953.

42. P. J. Flory, J. Am. Chem. Soc., 63, 3083 (1941).

43. S. B. Twiss, "Advancing Fronts in Chemistry," Reinhold Publishing Corp., New York, N.Y., 1945.

44. See, for example: P. G. de Gennes, "Scaling Concepts in Polymer Physics," Cornell University Press, Ithaca, New York, 1979.

45. D. Stauffer, "Introduction to Percolation Theory," Taylor Francis, London, 1985.

46. H. J. Herrmann, D. P. Landau, and D. Staufer, Phys. Rev. Lett., 49, 412 (1982).

47. H. J. Herrmann, D. Stauffer, and D. P. Landau, J. Phys. A: 
Math. Gen., 16, 1221 (1983).

48. M. Muthukumar, Macromolecules, 22, 4656 (1989).

49. J. E. Martin, D. Adolf, and J. P. Wilcoxon, Phys. Rev. A., 39, 1325 (1989).

50. W. Hess, T. A. Vilgis, and H. H. Winter, Macromolecules, 21 , 2536 (1988).

51. T. A. Vilgis, Progr. Colloid Polym. Sci., 90, 1 (1992).

52. A. Koike, N. Nemoto, M. Takahashi, and K. Osaki, Polymer, 35, 3005 (1994).
53. A. Hakiki, J. E. Herz, and G. Beinart, Polymer, 33, 4575 (1992).

54. J. Brabdrup, and E. H. Immergut, "Polymer Handbook," 3rd ed, John Wiley, New York, N.Y., 1989.

55. J. D. Ferry, "Viscoelastic Properties of Polymers," 3rd ed, John Wiley, New York, N.Y., 1980.

56. S. Onogi, T. Masuda, N. Miyanaga, and U. Kimura, J. Polym. Sci., Part A-2, 5, 899 (1967).

57. M. Takahashi, T. Takigawa, K. Yokoyama, and T. Masuda, $J$. Chem. Phys., 101, 798 (1994). 